Supporting Information for

\title{
High-Fidelity Replica Molding of Glassy Liquid Crystalline Polymer Microstructures
}

Hangbo Zhao,,$^{\dagger} \perp$ Jeong Jae Wie, ${ }^{\dagger,+}, \perp$ Davor Copic,,${ }^{\S}$ C. Ryan Oliver, ${ }^{\dagger}$ Alvin Orbaek White, ${ }^{\dagger}$ Sanha Kim, ${ }^{\dagger}$ A. John Hart ${ }^{* \dagger, \S}$

${ }^{\dagger}$ Department of Mechanical Engineering and Laboratory for Manufacturing and Productivity, Massachusetts Institute of Technology, 77 Massachusetts Avenue, Cambridge, Massachusetts 02139, United States

${ }^{\ddagger}$ Department of Polymer Science and Engineering, 100 Inha-ro, Nam-gu, Incheon 402-751, Republic of Korea

${ }^{\S}$ Department of Mechanical Engineering, University of Michigan, 2350 Hayward Street, Ann Arbor, Michigan 48109, United States

${ }^{\perp}$ These authors contributed equally to this work.

*Corresponding author: ajhart@mit.edu 


\section{Quantification of LCN micropillar actuation based on edge tracking algorithm}

To measure the thermally induced changes in the diameter and height of LCN micropillars we used image processing software written in $\mathrm{C}++$ based on the OpenCV library. The purpose of the edge tracking algorithm was to reduce bias introduced from manual measurements. The software sequentially measured the diameter of the pillar in each image along its height. First, the images were spatially registered using Adobe Photoshop, then the pillar diameters in the registered images were analyzed. This was done by thresholding the image (threshold value $=90$ ) and recording descriptors (average, standard deviation) of the pillar diameter along its height. To measure the height of a pillar, a CANNY edge finder was used to extract the pillar from the background using a minimum CANNY threshold value of 3 and then thresholding (threshold value $=125$ ) was applied to the resultant image. Then the height of the pillars was measured in the same manner as the diameter. Statistics were taken on each image to determine the average diameter and variation along the pillar.

During each heating and cooling cycle, 9 images were captured one minute apart at a fixed temperature. When analyzed, it was found that no significant change in pillar height occurred over this period and changes in dimension between heating and cooling were large enough that single image measurements would be accurate. The height values measured of homeotropic and polydomain structures were verified by using ImageJ (National Institute of Health) measurement manually to identify outliers. While the ImageJ measurements agree well with the automated measurements for the case of the polydomain height data it was found that the manual measurements were more accurate due to noise in the image. This was because the light changed between images such that no threshold value would separate the pillar from the background. 
a

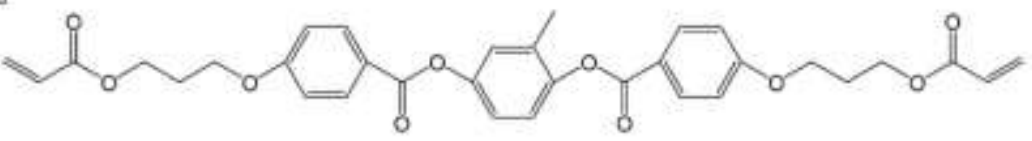

RM257

$\mathrm{b}$

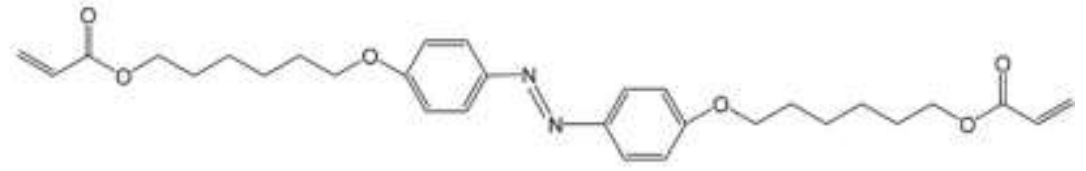

2-Azo

C

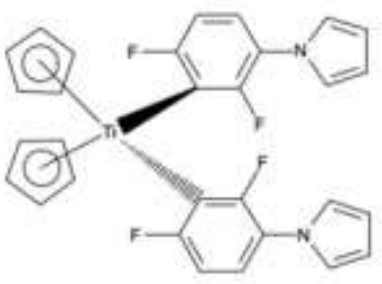

Irgacure 784

Figure S1. Chemical structures of mixture used to prepare LCN: (a) the monomer RM257, (b) 2Azo and (c) the photoinitiator I-784. 


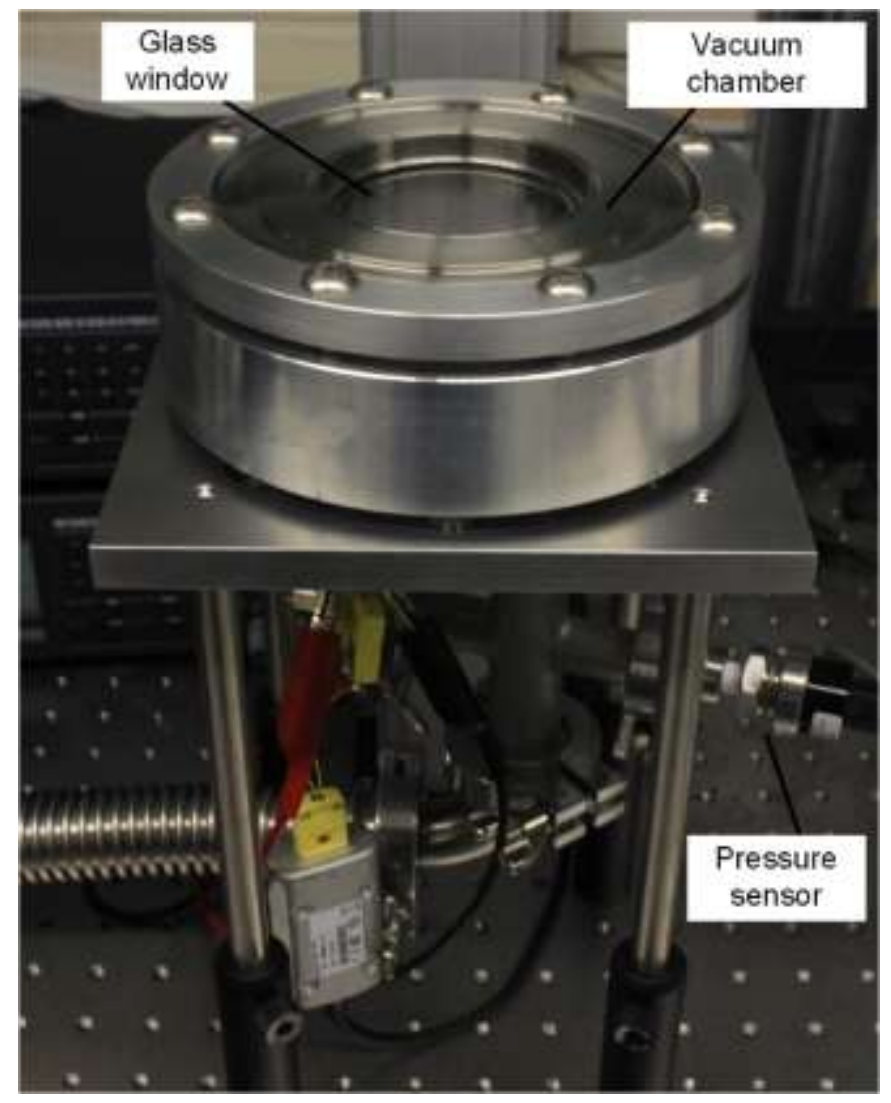

Figure S2. Vacuum chamber setup for replica molding of LCN microstructures, which encloses the stage assembly shown in Figure 1. 

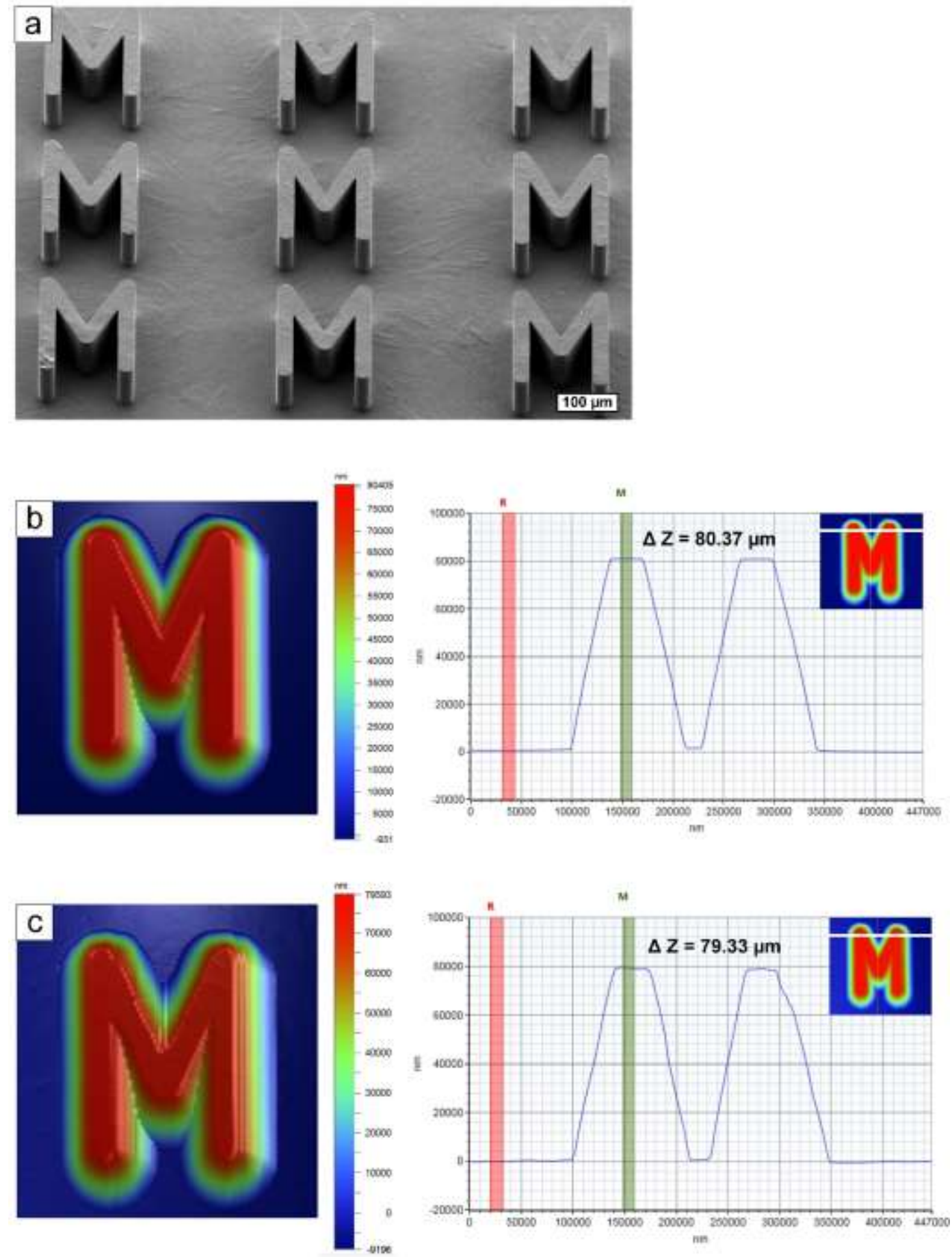

Figure S3. (a) SEM image of an array of letter "M" made of polydomain LCN. Surface profiling of a letter " $\mathrm{M}$ " on (b) the silicon master and (c) its polydomain $\mathrm{LCN}$ replica. The left sides in (b) and (c) are the 3-D scanning maps and the right sides are the 2-D line scan profiles near the top of the structures (position indicated by white lines in the top-right insets). The 2-D line scans were performed three times for both silicon master and the LCN microstructure. The sloped sidewall profiles artifacts arising from the tip-sample shape convolution. 
a

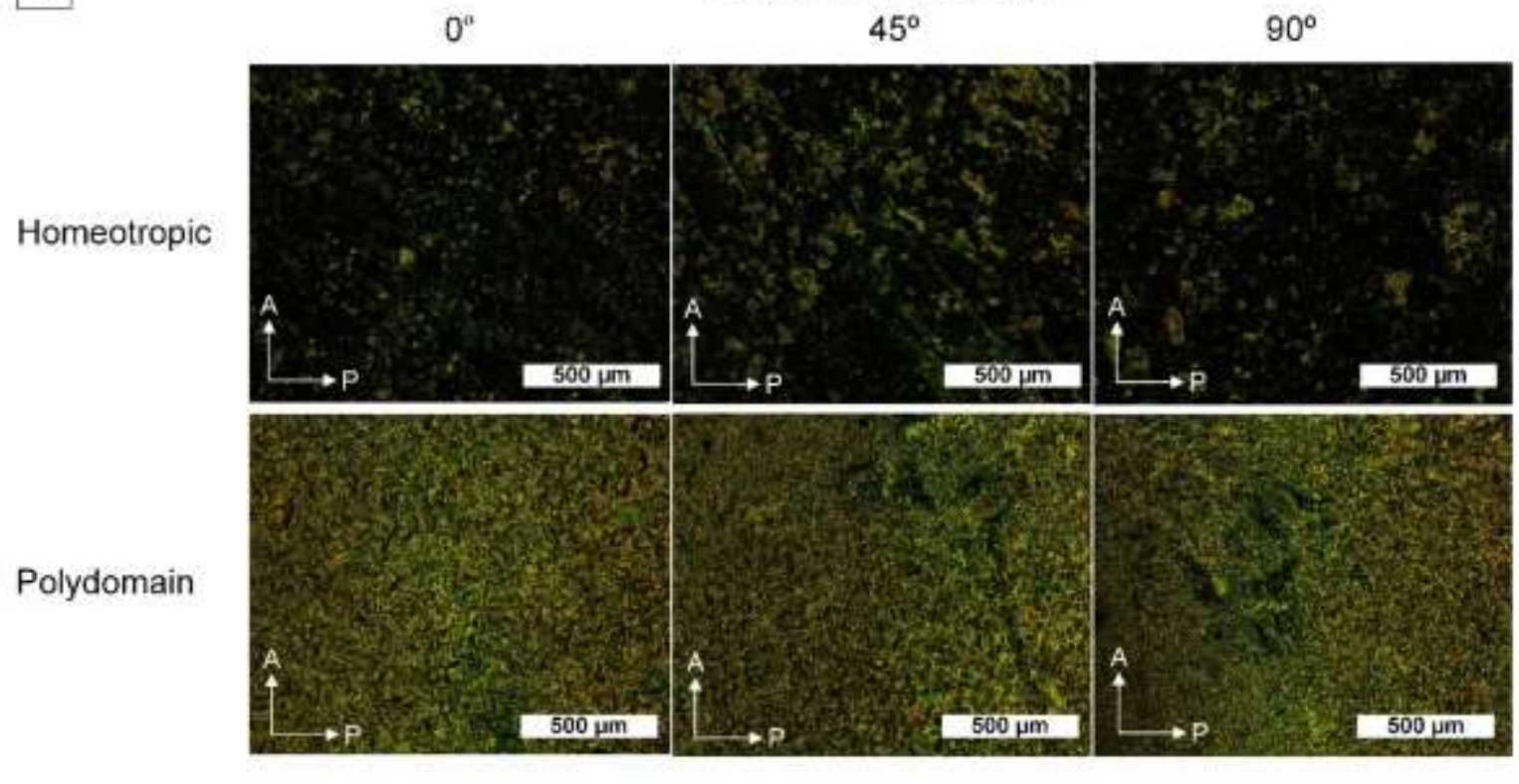

b

Homeotropic

Polydomain

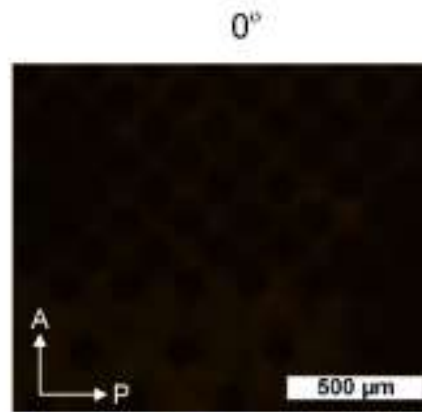

Sample Rotation Angle

$45^{\circ}$

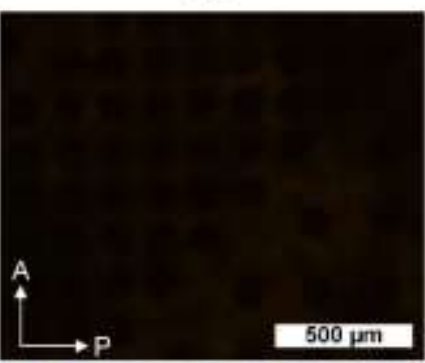

$90^{\circ}$
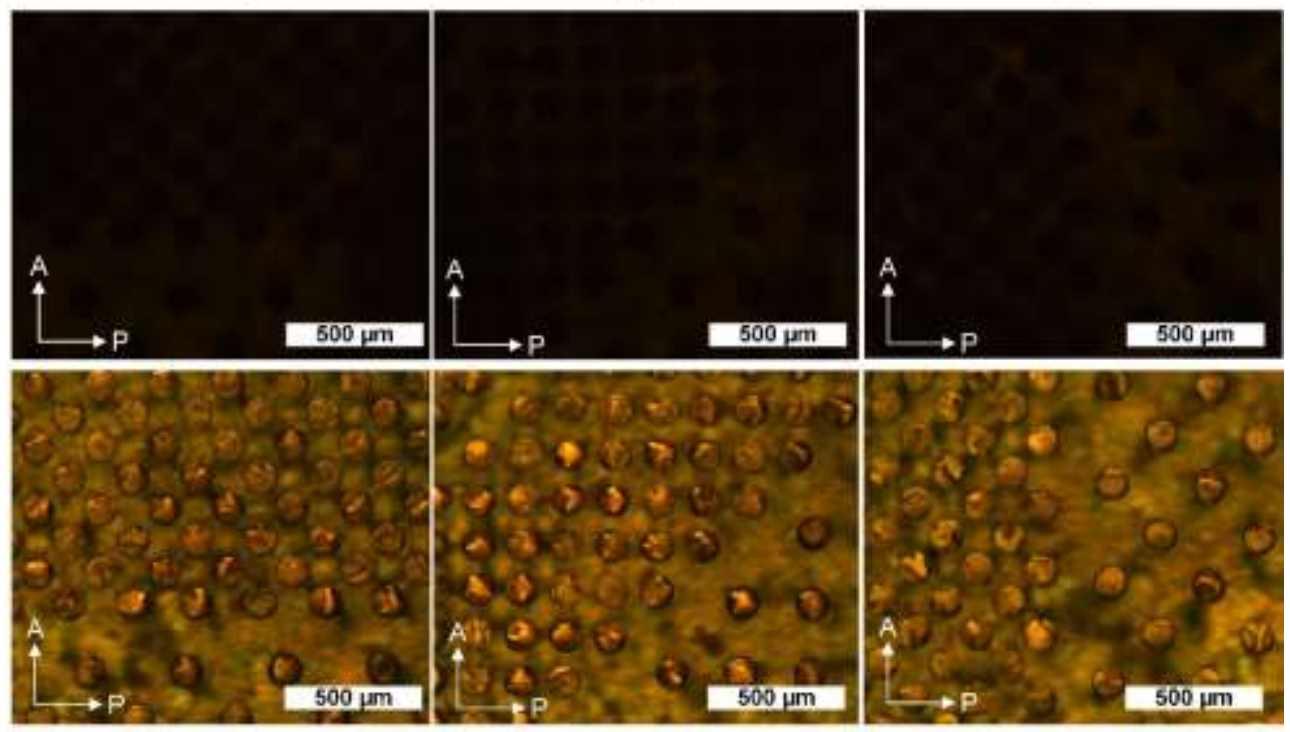

Figure S4. POM micrographs of (a) an LCN thin film and (b) an LCN micropillar array with homeotropic and polydomain order showing no dependence on sample rotation angles. 


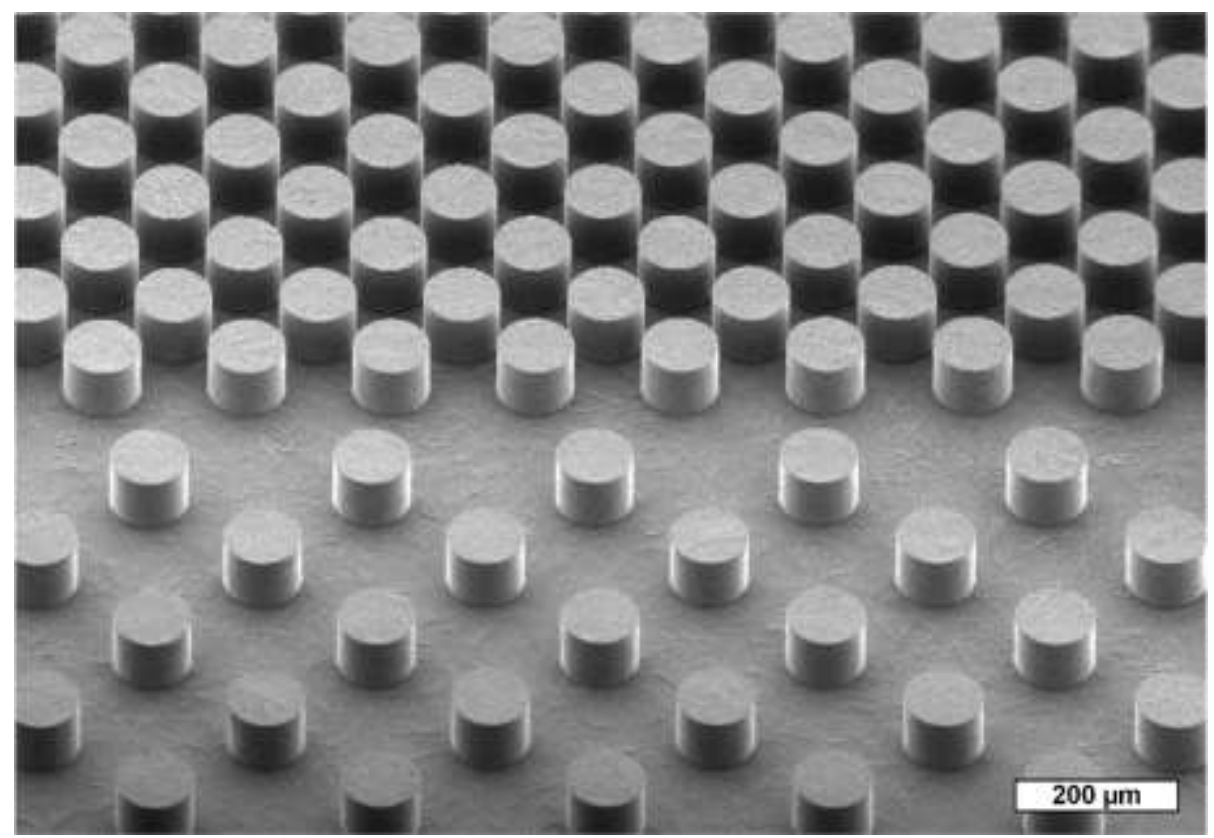

Figure S5. SEM image of polydomain LCN micropillar arrays. Homeotropic LCNs with the same structures were fabricated and compared using POM. 


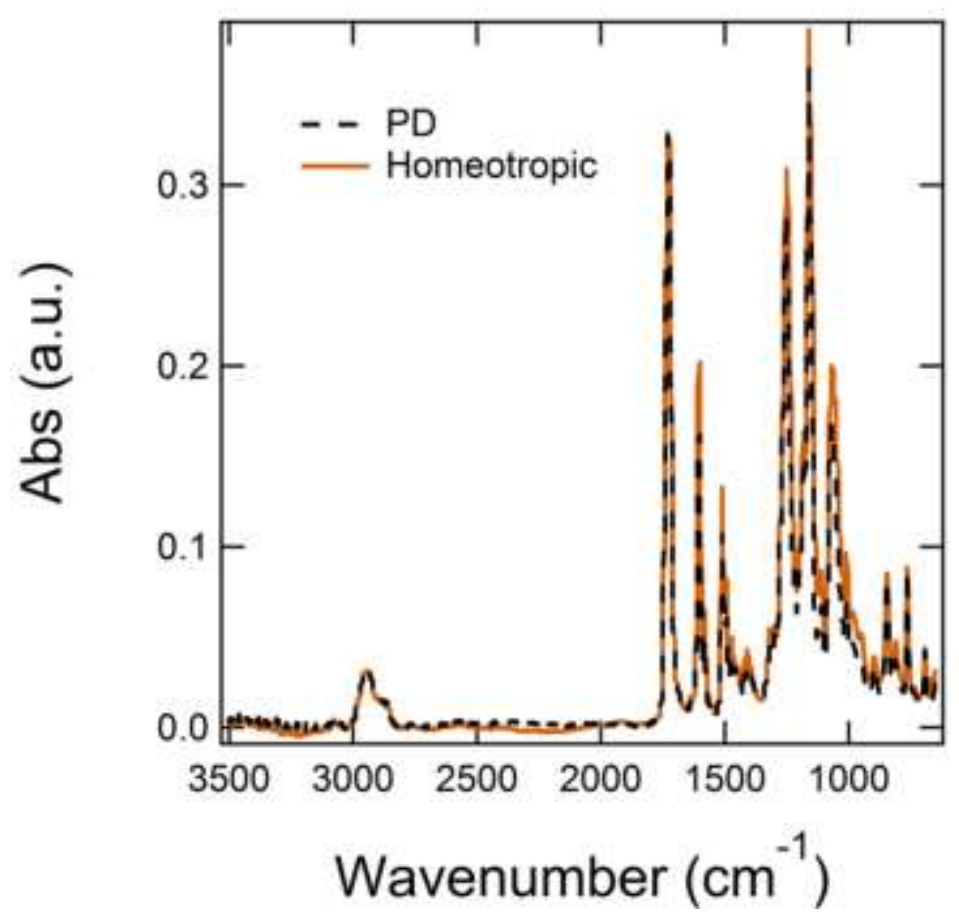

Figure S6. FT-IR spectra for polydomain (PD) and homeotropic LCNs. 

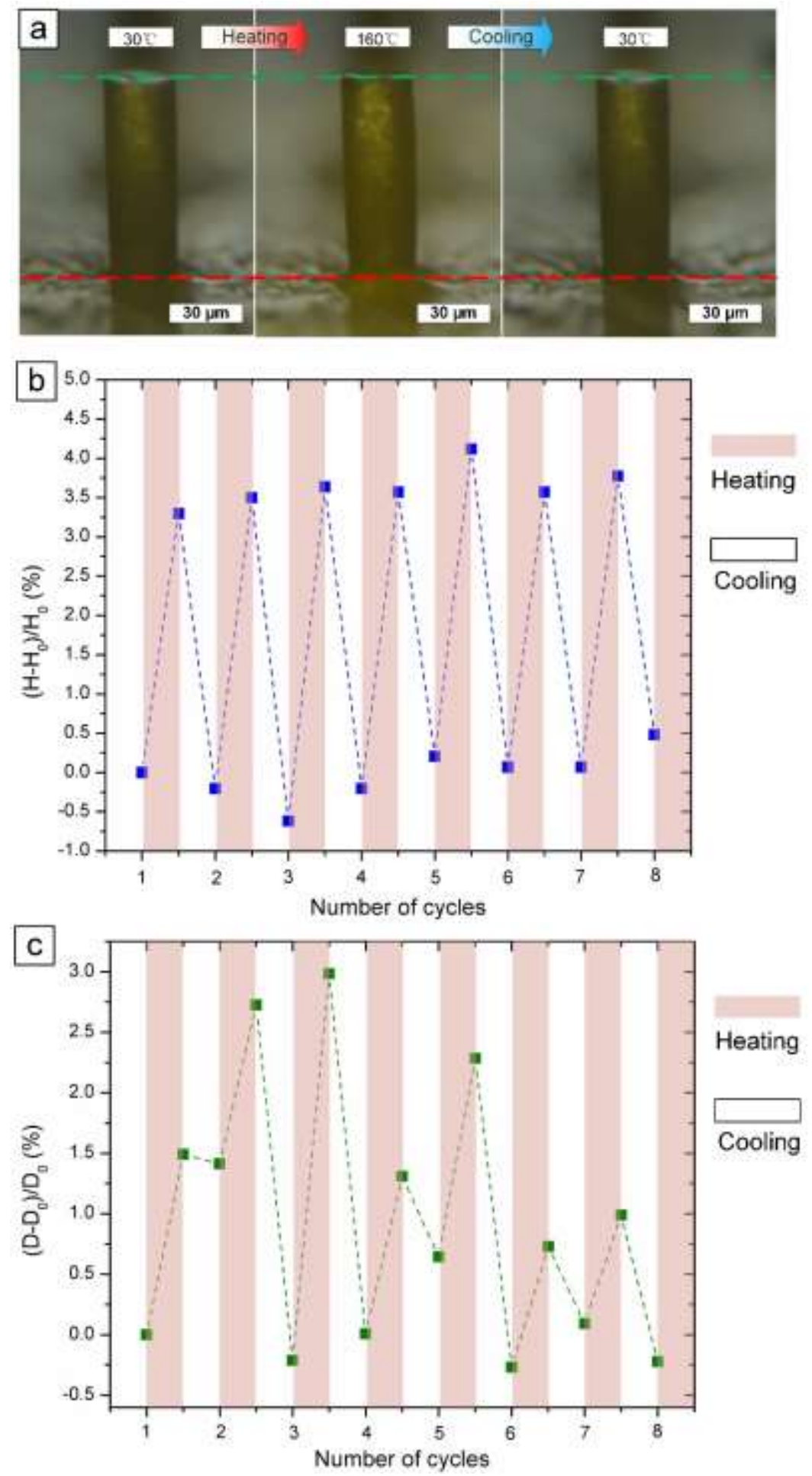

Figure S7. Thermal actuation of a polydomain LCN micropillar. (a) Side view of a $30 \mu \mathrm{m}$ diameter polydomain LCN pillar during heating and cooling. The three images are aligned at the base of the micropillar (indicated by the red dotted line near the bottom). The green dotted line near the top of the micropillar is aligned with the top surface of the micropillar in the left image $\left(30^{\circ} \mathrm{C}\right)$. Normalized height (b) and diameter (c) changes of the LCN micropillar during heating (to $160^{\circ} \mathrm{C}$ ) and cooling (to $30^{\circ} \mathrm{C}$ ) cycles. 


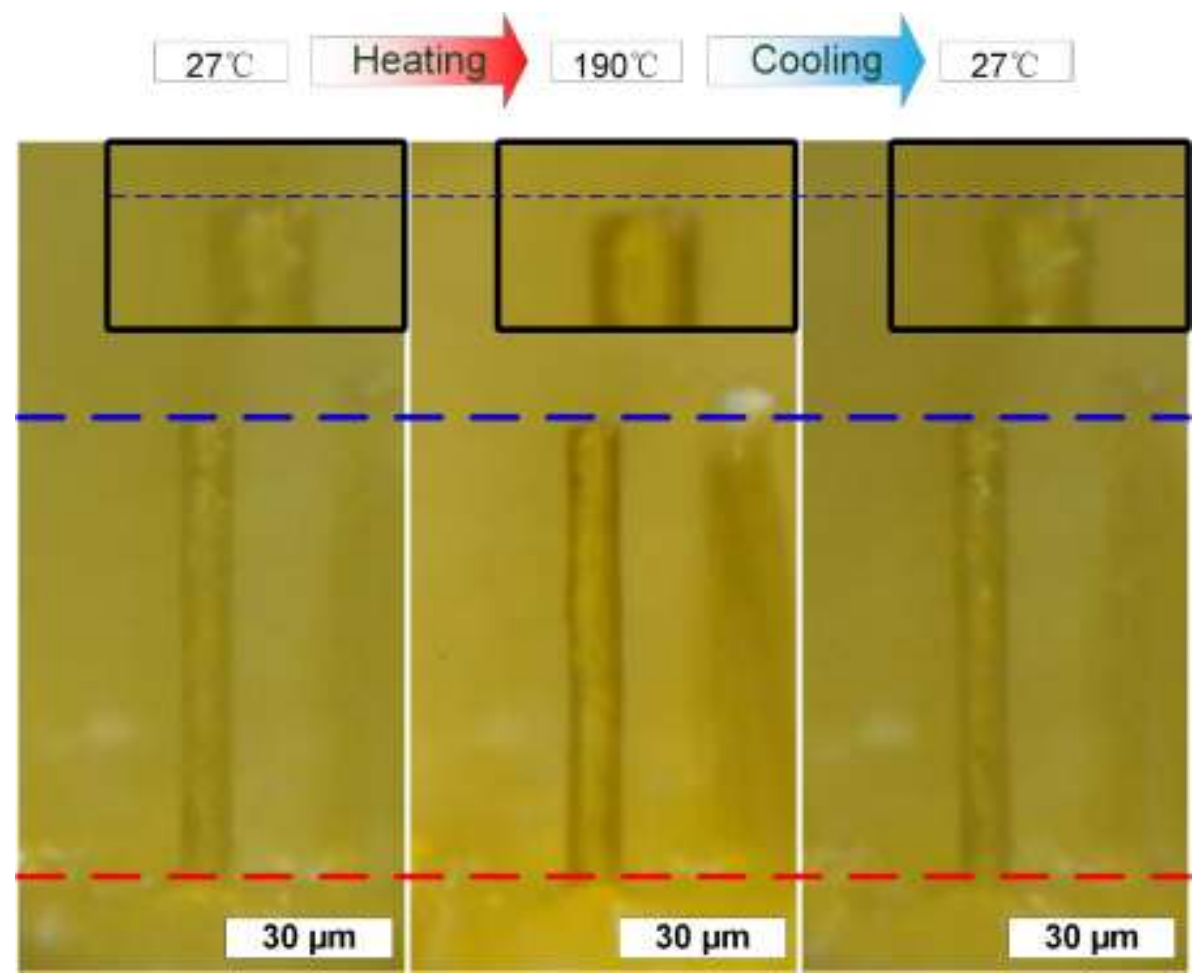

Figure S8. Thermal actuation of a $10 \mu \mathrm{m}$ diameter homeotropic LCN micropillar during heating (to $190^{\circ} \mathrm{C}$ ) and cooling (to $27^{\circ} \mathrm{C}$ ). The three images are aligned at the base of the micropillar (indicated by the red dotted line near the bottom). The blue dotted line near the top of the micropillar is aligned with the top surface of the micropillar in the left image. Insets are close-up images of the top of the micropillar. 